\title{
De la sociologie de « l'existence » à l'analyse des existants !
}

\section{Gilles Herreros}

\section{(2) OpenEdition \\ 1 Journals}

Édition électronique

URL : http://journals.openedition.org/pa/531

DOI : $10.4000 /$ pa.531

ISSN : 2273-0362

\section{Éditeur}

Université Lumière Lyon 2

\section{Édition imprimée}

Pagination : 16-29

ISBN : 1634-7706

ISSN : $1634-7706$

\section{Référence électronique}




\title{
De la sociologie de «l'existence » à l'analyse des existants!
}

\author{
Gilles Herreros
}

Université Lumière Lyon 2

Qu'est-ce que «l'existence»? Cette question est travaillée par les philosophes qui, depuis le socratique « connais-toi toi-même!", n'ont cessé de se pencher sur la signification de «l'existence » humaine. Du sujet pensant de Descartes, à l'existentialisme sartrien en passant par le "dasein » de Heidegger, les tenants de la philosophie ont traité de "l'être » et de ses conditions de déploiement. Depuis la fin du XIXe siècle, les psychologues et surtout les psychanalystes leur ont emboîté le pas, s'interrogeant, à leur tour, sur le «je »qu'ils ont pensé inconscient de lui-même, ayant du mal à être maître dans sa maison. Rimbaud avait lui aussi un avis comparable considérant que «je est un autre!», suggérant, par là-même, l'idée de l'ubiquité de "l'existence». Les sociologues, même s'ils ne sont pas aux premiers rangs de ceux qui ont exploré ce qu'exprime "l'existence», se sont également frottés à cette thématique. C'est d'ailleurs une habitude que la sociologie, fille de la philosophie comme toutes les sciences dites sociales, a toujours eu de retravailler les questions philosophiques premières. En les tournant, détournant, en les approchant différemment, les sociologues se sont saisis de nombre d'interrogations et de concepts philosophiques pour construire ce qu'ils ont appelé » leurs objets ». Sans prétendre fournir une revue exhaustive de la littérature sociologique portant sur "l'existence», quelques repères peuvent néanmoins être évoqués. Marx (annexé aussi bien par les sociologues que les économistes ou les philosophes) dans L'idéologie allemande, affirmait que les conditions matérielles de "l'existence » déterminaient la conscience. Avec cette idée, il donnait le «La» que les sociologues reprendront à leur compte : l'être est le produit de son histoire, de son environnement, lesquels déterminent ce qu'il fait et, au final, ce qu'il est. Fort de cet héritage intellectuel (et de nombreux autres évidemment) les sociologues étaient mis sur la voie ; la question de "l'existence » pouvait être placée au rang de leurs préoccupations. Que ce soit avec les travaux de G.H. Mead Le moi, le soi et la société ou bien avec les recherches beaucoup plus récentes de V. de Gaulejac (une des figures de proue de la sociologie clinique) réfléchissant sur Qui est "je» ou d'A. Touraine, qui dans sa Critique de la modernité invite à la revitalisation du « sujet », les sociologues, selon des voies très différentes, ce sont fréquemment confrontés à « l'existence ». Toutefois, il convient de noter que cette confrontation n'a, le plus souvent, pas transité par 
la notion explicite «d'existence ». En effet, c'est via le «moi » (Mead), le «sujet» (Touraine, 1992), le «je» (de Gaulejac, 2009), l'«individu» (Martucelli, De Singly, 2009), la " personne »(Boltanski, Thévenot, 1991), l' « ego » (Kaufmann, 2001), que la question de «l'existence » a été approchée.

Toutefois, récemment (2011), dans un long article de la revue SociologieS, D. Martucelli s'est proposé, en tant que sociologue, à une confrontation directe avec le terme "d'existence»: "Une sociologie de l'existence est-elle possible?» interroge-t-il. C'est à partir de ce texte que nous voudrions introduire notre propos. Nous allons tout d'abord suggérer que cet article de D. Martucelli, malgré tous ses mérites, illustre la difficulté que les sociologues ont à se démarquer des débats philosophiques pour penser "l'existence » comme «objet» sociologique. A prendre appui sur la philosophie pour montrer la plus-value de l'approche sociologique ( $\mathrm{du}$ moins celle dont Martucelli se réclame), la démarche débouche, je crois, sur un affaiblissement $\mathrm{du}$ raisonnement philosophique sans que le détour sociologique ne parvienne à compenser cette perte. Ensuite, je proposerai l'idée, selon laquelle, il ne peut y avoir de supplément cognitif en provenance des sciences sociales en général et de la sociologie en particulier sur cette thématique, si ne sont pas opérées deux inflexions. La première, qui fera l'objet du second paragraphe de ce texte, consiste à glisser du terme "d'existence » à celui "d'existants ». La seconde suggère de s'attacher non plus à "l'existence » mais aux "modes » d'existence ; ce sera l'objet du troisième point abordé.

Ces deux glissements nous sont proposés par deux auteurs dont nous peinons à qualifier la discipline à laquelle ils se rattachent. Le premier, $\mathrm{P}$. Descola, est, du point de vue du statut, professeur d'anthropologie au Collège de France mais ses travaux sur les formes d'organisations sociétales des différentes communautés humaines peuvent aisément être rapprochés de la réflexion sociologique dès lors que l'on comprend cette discipline comme l'étude des règles que les humains se donnent pour vivre les uns avec les autres. Le second, B. Latour, est sociologue rattaché à l'Institut d'Etudes Politiques de Paris, mais ses travaux relèvent indistinctement, de la philosophie des sciences, de l'anthropologie des modernes, de la sociologie de l'acteur réseaux et plus généralement de ce qu'il appelle les «humanités scientifiques » (2010). Descola et Latour, dialoguant à distance depuis de nombreuses années, sont moins les représentants de disciplines distinctes que des tenants de l'univers des sciences sociales ET $^{1}$ humaines. Les enfermer dans une case académique ne serait pas pertinent; cet emprisonnement n'est pas nécessaire pour approcher ce qu'ils apportent à la réflexion sur « l'existence » 2 et qui semble relever d'un véritable changement de paradigme.

1 Les majuscules affublées à la conjonction de coordination « et » renvoient, comme je l'évoquerai en conclusion de ce texte, à la distinction que A. Piette suggère d'établir entre sciences sociales (relationnistes) d'une part et sciences humaines d'autre part.

2 Nous allons nous passer de guillemets désormais pour parler de la notion d'existence. 


\section{SOCIOLOGIE DE L'EXISTENCE : UNE CONTINUITE PARADIGMATIQUE}

Dans l'article mentionné plus haut, D. Martucelli, dont on peut considérer $\mathrm{qu}^{\prime}$ il est un sociologue issu de la tradition tourainienne (filiation dont il s'est à la fois émancipé sans pour autant s'en éloigner, ni s'y opposer), se propose de donner à voir qu'une sociologie de l'existence est possible. Pour ce faire, il s'engage dans deux directions. La première consiste à reprendre, en des termes intelligibles pour les sociologues, quelques-uns des débats philosophiques traitant de l'existence pour montrer qu'ils sont le plus souvent tournés vers l'ontologie et qu'ils ne permettent donc pas de penser les dimensions contemporaines de l'existence. La seconde orientation, supposée dépasser la limite du raisonnement philosophique, relève d'une forme de sociologisme classique où il est réaffirmé que ce sont les conditions matérielles (sociales) d'existence qui produisent sinon la conscience au moins les opportunités de déploiement de l'individu. Ces deux points méritent d'être repris.

Dans un savant et très long développement, D. Martucelli revient sur les principaux apports de la philosophie en matière de réflexion sur l'existence. On y trouve ainsi convoqués nombre de concepts produits par les philosophes pour approcher la question. Pascal considérant que l'existence doit conduire à éviter de se perdre dans les divertissements, Heidegger et son "dasein » qui place le souci de son «être » au centre des préoccupations de l'homme, lequel, confronté à l'épreuve de l'angoisse de vivre, doit s'acquitter de la « besogne » d'être, conception que ne partage pas son collègue suédois Kierkegaard convaincu que c'est dans l'expérience du monde que l'existence s'abime ou non. C'est sur Sartre que s'arrête plus longuement le texte en indiquant d'une part que " tous les chemins » partent de son existentialisme et que d'autre part la pensée sartrienne a pu évoluer considérablement au fil du temps se déplaçant depuis l'affirmation d'une forme de liberté ontologique et anhistorique de tout homme pour déboucher progressivement sur des dimensions plus matérialistes et historiques de l'expérience humaine. En réorganisant le dialogue entre Heidegger, Sartre, Kierkegaard, mais aussi quelques autres (Pascal, Bubber, Levinas, Camus, Habermas...), puis en réexaminant ce que pourraient être les rapports entre existence, essence, expérience, déterminisme ou liberté ontologique, conflit des consciences, existence en soi ou pour soi, Martucelli entend montrer que les philosophes et en particulier Sartre ne sont pas parvenus à penser la vie sociale. Heureusement, les sociologues tels que Simmel et Mead (aidés tout de même par Kierkegaard "premier philosophe de la modernité » selon Martucelli) avec leur approche de la modernité sont parvenus à suggérer que la question de l'existence ne pouvait être examinée sans la prise en compte de la vie sociale. Cela étant, l'auteur de l'article débouche sur le constat qu'entre la philosophie de l'existence et les sociologies existentialistes le débat s'est 
enfermé dans une impasse celle d'un ek-sistere (être en dehors de soi) qui ne serait abordé que du point de vue de la problématique dualiste de la nécessité et de la liberté.

$\mathrm{C}^{\prime}$ est à cet endroit qu'il situe le début de sa proposition. Une sociologie de l'existence d'un nouveau type permettrait de sortir du dialogue bloqué entre philosophie et sociologie à la condition de procéder à quatre déplacements. Le premier suppose une inflexion historiciste prenant en compte l'expérience de la modernité laquelle permettrait de comprendre autrement ce qu'est l'existence. En d'autres termes, comment passer de la réflexion concernant la condition humaine à celle qui traite de la condition moderne. Le second passerait par la prise en compte de l'élargissement des états existentiels qu'occasionne la modernité et qui conduit les individus à faire massivement l'expérience de l'isolement, de la solitude, du chômage, de leurs fragilités, de leurs sentiments d'étrangeté. En somme, comment passer de l'étude des existentiaux anthropologiques à la connaissance de l'individuation. Le troisième déplacement que propose Martucelli consiste à cerner les différents esprits existentiels des époques en soulignant notamment qu'il convient de glisser de «la question existentielle contemporaine de l'anxiété herméneutique »-quel sens donner à l'existence- vers l'anxiété énergétique (sur quoi m'appuyer pour agir)" ce qui revient, selon lui, à faire de l'interrogation sur ce que sont les supports de l'existence une question sociale. Enfin, dernier des déplacements suggérés, il convient pour une sociologie de l'existence de saisir la «dynamique des réalités socio-existentielles contemporaines ». Ces réalités concernent les épreuves qu'occasionne l'expérience de la parenté biologique ou non, l'expérience de la mort que la modernité entend faire reculer sans cesse plus et que l'on voudrait voir administrer dans un cadre éthico-thérapeutique, la confrontation à la fin de vie qui devient toujours plus insupportable. En d'autres termes, la modernité nous confronte à des épreuves sociales qui n’ont jamais été aussi fortement des épreuves existentielles.

Le programme que Martucelli assigne à une sociologie renouvelée de l'existence, pour problématiser et très cadré qu'il soit, me semble présenter deux limites majeures. La première réside dans le fait que les quatre directions proposées ne me paraissent pas nous faire sortir de l'impasse qu'a occasionné le débat entre philosophie et sociologie et qu'il résume lui-même sous le vocable de la dialectique de la nécessité et de la liberté. Ce programme en quatre points me semble en effet largement relever d'une description sociologique de la nécessité qu'imposent aux individus et aux collectifs les impératifs existentiels de la modernité. Du coup se trouvent assez largement écrasées toutes les questions sur ce que l'existence peut posséder de transhistorique et que les philosophes mieux que les sociologues savent approcher. La deuxième difficulté me paraît résider dans l'absence de renouvellement conceptuel que cette sociologie de l'existence semble receler. En effet sans 
concepts d'un nouveau genre comment prétendre sortir de débats qui pourraient être qualifiés de séculaires?

Nous ne prétendons évidemment pas substituer une sociologie dont nous aurions la paternité à celle qu'avance Martucelli. Plus simplement, nous pensons que s'il est question de changer de direction d'analyse pour penser une sociologie de l'existence, ce sont de tout autres déplacements qu'il faut suggérer; des déplacements permettant d'entrevoir un changement de paradigmes dans les sciences sociales ET humaines. Les travaux de Descola et ceux de Latour nous en fournissent l'esquisse.

\section{UNE ANTHROPOLOGIE DES ONTOLOGIES MULTIPLES}

P. Descola, titulaire de la chaire d'anthropologie de la nature au Collège de France, développe, depuis sa thèse (réalisée sous la responsabilité de C. LeviStrauss) une approche des cultures amérindiennes. Il débouche sur l'idée que ces populations élaborent des types de rapports au monde très différents de ceux pratiqués dans la modernité contemporaine par les Occidentaux. Pour ces derniers une discontinuité nette est établie entre les entités humaines et les autres existants. En effet selon les modernes, les végétaux, les minéraux, les animaux ne sont pas dotés d'une capacité de conscience et leur physicalité les distingue irrémédiablement des humains. Ainsi la culture est-elle située du côté des entités humaines pendant que les autres existants sont considérés comme relevant exclusivement de la nature. Si l'homme existe en tant qu'homme c'est qu'il est parvenu à s'extraire, à s'arracher de l'état de nature par sa capacité réflexive. Dans une tradition intellectuelle qui remonte aux classiques de la philosophie (Descartes, Locke, Leibniz et bien de leurs prédécesseurs) les modernes se sont donc convaincus de leur discontinuité avec tous les autres existants. Ainsi la séparation entre nature et culture s'estelle imposée comme la clef de leur rapport au monde. La culture est supérieure à l'état sauvage de la nature dont les hommes se sont émancipés ; cette dernière peut donc être enrôlée, exploitée, asséchée, dévastée pour les besoins humains. En toute logique, dès lors que l'existence est définie comme une propriété qui passe par l'étude exclusive de l'esprit humain et l'enveloppe physique qui le contient, tout ce qui relève de l'environnement passe au second plan. Les variables extérieures qui pourraient être explicatives de ce qui fait exister l'homme peuvent avoir leur importance mais elles ne relèvent pas de ce qui est essentiel : l'entité humaine ne peut être assimilée aux autres entités du monde. En ce sens, et en ne forçant pas le trait de façon excessive, il est possible d'affirmer que les travaux classiques des philosophes, des sociologues et plus largement des tenants des "humanités », se sont focalisés sur l'étude de l'existence humaine en la bordant avec des frontières culturelles. Que ce soit pour étudier le dasein, l'identité en soi ou pour soi, qu'il se soit agi d'historiciser ou non l'ontologie ou bien encore, comme le fait 
Martucelli, que l'effort soit porté sur une sociologisation des traits d'une modernité contemporaine qui redessine les formes d'existence, l'existence est toujours placée du côté des entités humaines culturalisées.

Pourtant, il est d'autres façons possibles de penser le rapport au monde et du coup de s'interroger sur ce qu'est le fait d'exister. Ce "naturalisme » des modernes, selon la terminologie qu'utilise Descola, qui conduit à la séparation entre les entités humaines et tous les autres existants, n'est pas le seul vecteur possible pour établir un rapport au monde. Trois autres conceptions sont inventoriées par Descola : l'animisme le totémisme et l'analogisme.

L'animisme, dont on peut retrouver des formes aussi bien chez les indiens d'Amazonie que chez les Kasua du Grand Plateau de Papouasie-Nouvelle Guinée, se traduit par une prise en compte des physicalités différentes que revêtent par exemple les entités animales et les entités humaines mais ce qui est souligné depuis cette conception c'est avant tout la similitude des intériorités. Ainsi, les animaux sont considérés comme ayant une substance interne semblable à celle des humains ; seuls leurs traits externes varient. En somme, tout ne serait qu'une affaire de peau. Il y a discontinuité des corps mais similarité des âmes. Sans entrer dans le détail des principes animistes qui, par exemple, attribuent des capacités de métamorphose changeantes selon les individus (si un chamane peut se faire jaguar tous les humains ne peuvent avoir cette faculté), on peut observer que dès lors qu'une continuité des intériorités est établie entre les humains et les animaux la question des équilibres et des coupures entre le « règne animal » et la présence humaine n'a plus les mêmes contours que chez les naturalistes. Pour le dire trivialement, penser l'existence comme une substance qui ne soit pas proprement humaine reconfigure la question d'une sociologie des existants. Les frontières ne sont plus « sociales », ni « historiques », comme les désignent les sociologues.

Le raisonnement est de même facture pour ce qui relève du totémisme. Là où les communautés humaines relèvent d'un rapport au monde totémique (chez certains aborigènes d'Australie mais aussi dans le sud-est des EtatsUnis, en Amérique du Nord et dans nombre de communautés -Mangarrayi, Yangman, Chickasaw, Potawatomi, Ojibwa, Cree, Penobscot...précise P. Descola en appui sur une volumineuse littérature), chaque individu se trouve associé à une entité non humaine avec laquelle il entretient une continuité externe et interne (physique et mentale). L'ontologie totémique, que ce soit sous une forme très marquée ou bien à l'état d'esquisse, suppose l'existence de continuités entre les humains et les entités du monde animal. Ainsi, la nourriture consommée peut être la même que celle qu'ingère tel ou tel animal (le renard rouge ou le puma), les territoires de chasse sont partagés avec les animaux lesquels peuvent être considérés comme propriétaires desdits territoires dont les hommes n'ont que l'usufruit obtenu après une forme de pacte passé avec eux.

Pour ce qui relève enfin de ce que Descola nomme les "vertiges de l'analogisme », dont il y eut des traces dans l'Europe du XVIe siècle mais aussi 
et surtout dans l'Inde brahmanique, en Afrique de l'ouest, en Chine ancienne, dans la zone andine et le Mexique précolombien, ce qui est notable c'est la forte hiérarchisation des entités. La classification verticale en classes, en castes, en fonctions en empilement de puissances et de divinités se retrouve dans toutes ces aires historiques ou géographiques; il en est de même de la métempsychose. Les individus réapparaissent sous une forme animale, l'âme d'un parent peut se retrouver dans la mare de la propriété familiale, ou s'incarner dans un arbre.

En d'autres termes, là où les naturalistes que sont les modernes, découpent le monde entre nature et culture, là où l'animalité humaine a été domptée grâce à la culture, les trois autres ontologies animiste, totémique et analogique suggèrent des liens fort différents entre toutes les entités du monde, qu'elles soient minérales, végétales, animales, en prenant en compte leurs intériorités spirituelles ou leurs extériorités physiques. Les découpages (ou absence de découpages) et les hiérarchies (ou absence de hiérarchie) auxquels procèdent les différentes communautés humaines existantes ou ayant existé, débouchent sur des visions du monde, et plus globalement du cosmos, très différenciées. A partir d'un cadre d'analyse prenant en compte ce constat, on imagine aisément que les frontières de l'existence se déplaceront fortement selon l'ontologie de référence. Du coup, une sociologie de l'existence qui se proposerait d'enrichir la philosophie heideggerienne, sartienne, camusienne, habermassienne en continuant de ne penser les existants que dans le cadre naturaliste ne constitue qu'un déplacement minime dont il n'est pas certain qu'il soit un approfondissement. Que la modernité contemporaine conduise à l'émergence d'interrogations nouvelles percutant les liens entre individu et société (comment accompagner la fin de vie, y-a-t-il un usage éthique de l'euthanasie, peut-on concevoir la Procréation Médicalement Assistée, la gestation pour autrui ... ?) et que les sociologues suggèrent, dès lors, que la réflexion sur l'existence ne peut plus ne pas être sociologique, possède une certaine pertinence mais nul doute que les philosophes spécialistes du " dasein », discutant depuis des décennies la profondeur des distinctions entre l'être et le non-être trouveront le «déplacement» plus anecdotique que fondamental.

A l'ère de «l'anthropocène » (nouvelle ère géologique au sein de laquelle ce sont désormais les activités humaines qui déterminent décisivement l'avenir $\mathrm{du}$ système terrestre), pour reprendre une formule de la stratigraphie, la question de l'existence mérite sans doute d'être réinterrogée par les spécialistes des sciences sociales à partir d'un matériel théorique nouveau. Pour changer la société il faut faire de la sociologie autrement (Latour, 2006), changer de paradigmes. C'est ce que propose, je crois, Bruno Latour dans un texte comme «Enquête sur les modes d'existence » (2012). 


\section{L'ANTHROPOCÈNE ET LES MODES D'EXISTENCE}

Tout comme il n'était pas question de rendre compte de la somme monumentale que constitue le travail de P. Descola, il ne peut être ici envisagé de présenter les abondants et importants travaux que Bruno Latour produit depuis plus de trois décennies. Je vais donc me contenter d'évoquer l'ouvrage publié en 2012 sous le titre de "Enquête sur les modes d'existence ». Dans cet ouvrage l'auteur esquisse ce qui nous parait être un chemin possible pour les sciences sociales ET humaines en matière de réflexion sur l'existence, chemin débarrassé de tout sociologisme. C'est tout d'abord sur le titre qu'il convient de s'arrêter. A lui seul il indique l'orientation latourienne; elle est bien éloignée d'une sociologie de l'existence telle que rappelée dans le premier paragraphe de ce texte. A sa lecture, trois remarques s'imposent. Tout d'abord, en ne spécifiant pas la nature de l'enquête qu'il engage (on ne sait pas, en effet, si elle sera anthropologique, philosophique, sociologique, politique, éthique...), l'auteur nous indique son indétermination disciplinaire, ou son indiscipline. Si le sous-titre "Anthropologie des modernes » laisse entendre que le propos sera plus maussien que durkheimien, l'examen du texte permet de constater que l'enquête conduite n'est ni sociologique, ni philosophique, ni anthropologique, mais tout cela à la fois, et plus encore. En effet, les investigations qu'il entreprend sont non seulement d'ordre sociologico-philosophico-anthropologico-... mais elles relèvent aussi et plus généralement de ce qu'il nomme les «humanités scientifiques $»^{3}$. Pour comprendre le monde, depuis lesdites « humanités scientifiques », nul besoin, selon B. Latour, de coincer l'enquête dans des cercles disciplinaires bornés par des sciences séparées qui passent leur temps soit à s'ignorer soit à se disqualifier réciproquement -sciences dures contre sciences molles, psychologie contre sociologie, sociologie contre philosophie, philosophie contre anthropologie et peut être aussi sciences sociales contre sciences humaines ... Le spécialiste des humanités scientifiques doit pouvoir faire feu de tout bois sans s'encombrer de frontières disciplinaires lesquelles vont toujours de pair avec des douanes méthodologiques et conceptuelles, avec des douaniers toujours prêts à voir partout des contrebandiers. S'interroger sur les modes d'existence des modernes est une question si complexe qu'elle autorise bien, a minima, une entorse aux carcans académiques.

La seconde remarque qu'inspire le titre de cet ouvrage porte sur le fait que B. Latour n'entende pas traiter de l'existence mais bien plutôt des « modes d'existence ». Avec cette formulation il laisse comprendre que l'existence en

3 Pour Latour, les humanités scientifiques ne relèvent pas de «La Science » entendue au singulier et avec un « $S$ » majuscule. Elles s'apparentent plutôt à ce qu'il nomme l'activité scientifique. Là où «La Science » fonctionne comme une sorte de stock de savoirs exerçant un contrôle sur le juste et le faux, l'activité scientifique est plus erratique s'accommodant d'un mouvement brownien, chaotique ou pillage et bricolage participent de la production de faits dont la vocation est d'être controversés. 
elle-même est moins intéressante que les modes à partir desquelles elle se décline. La terminologie laisse ainsi entrevoir que son projet est résolument asubstantialiste. Ce n'est pas l'existence qui l'intéresse en tant que substance mais ses modes de déclinaisons. En d'autres termes, et en jouant avec les mots, on pourrait dire que, pour Latour, ce qui fait le fond de l'existence (à supposer que l'existence est un fond) c'est sa forme. La forme devient le fond.

Enfin, troisième remarque, le titre nous indique que l'enquête conduite ne concernera que les « Modernes ». Bien sûr, Latour nous a expliqué par le passé (1991) que les contemporains se prévalant de modernité n'avaient jamais été modernes. Faut-il voir là une marche arrière ou un reniement ? La majuscule dont il affuble les « Modernes » nous met sur la voie. Les « Modernes » se sont auto-proclamés tels pour se démarquer des primitifs, des fétichistes (ceux-là même qu'ils ont colonisés en prétendant leur apporter la civilisation) mais rien ne prouve qu'ils soient si modernes! N'ont-ils pas eux aussi leur fétiches «faitiches" (Latour, 1996) ? En outre, les «Modernes» ne sont pas si nombreux ; ils ne peuvent prétendre incarner l'humanité dans son ensemble. En réglant sa focale analytique sur eux seuls et sur les relations qu'ils entretiennent avec les autres existants du monde, Latour nous suggère que leurs modes d'existence ne sont que des modes parmi d'autres. Dans le vocabulaire de P. Descola on pourrait indiquer que B. Latour reprend à son compte l'idée qu'il y a une pluralité de relations au monde, une pluralité d'ontologies, et que celle des naturalistes (les « Modernes") n'est pas la seule possible.

Au-delà du titre et de ce qu'il suggère, l'ouvrage défend une thèse dont quelques lignes de force peuvent être dégagées car elles permettent de cerner comment la question de l'existence peut être appréhendée4 depuis une perspective latourienne.

L'ouvrage pose d'abord le contexte de la réflexion en donnant la parole à Gaia (la terre donc). Elle interpelle les «Modernes » : si vous faites « la sourde oreille en ignorant l'anthropocène » votre destruction est promise leur dit-elle. «Moderniser ou écologiser, il (vous) faut choisir!». Jusque-là, rappelle Latour, « les Modernes » ont pensé leur habitat, leur oikos, à partir des règles (nomos) de l'éco(nomie). L'habitat qui en a résulté est devenu impraticable ou le sera bientôt -réchauffement du climat, déforestation, pollution, disparition de la couche d'ozone... pour n'en rester qu'aux questions environnementales actuelles lesquelles précèdent la raréfaction de l'eau et son probable cortège de migrations massives, de conflits de territoires etc. Il faut donc que les «Modernes» imaginent le réaménagement de leur habitat selon d'autres règles, celles de l'écologie. Coincés entre la dystopie (le cauchemar de l'économisation forcenée) et l'utopie (d'une écologisation difficile), les "Modernes" doivent repenser leurs rapports à ce qui les entoure. Là se trouvent les conditions de possibilité du maintien de leur existence. Des «préliminaires de paix» doivent donc être engagés avec Gaïa. En

4 Pour une compte rendu plus complet de cet ouvrage cf. Herreros 2014. 
chercheur/diplomate, B. Latour offre ses services pour ouvrir la négociation. En premier lieu il propose de liquider les sociologies traditionnelles et les impasses auxquelles elles conduisent. Ainsi, il se débarrasse de la séparation entre l'objet et le sujet, de la distinction culture/nature, de la coupure entre la science et le politique, de l'opposition entre faits et valeurs. Une sociologie de l'existence qui se décline (à la façon de celle présentée dans la première partie de cet article) sous la forme d'une réflexion qui vise à ne penser les rapports entre individus et société que sous l'angle de leurs interactions et qui part à la recherche des déterminations qu'elles soient structurelles, historiques, sociales ou bien qui mobilise le souci de prendre en compte l'expérience sensible des individus (celle de la domination, de l'humiliation, de l'échec, de la fin de vie...) ne permet pas de saisir la question de l'anthropocène. Les conditions d'existence de tous les existants sont désormais liées aux possibilités qu'ils auront de tenir ensemble dans des rapports qui ne soient pas de destruction. C'est donc à une réhabilitation des existants que les "Modernes » utilisent comme des ressources à détruire, des ressources consommables, de simples supports, qu'il faut procéder. Pour le dire encore autrement les sciences sociales devraient avoir comme urgence non plus un programme d'analyse des déterminations multiples, des actions réciproques, qui font que les individus et les sociétés sont ce qu'ils sont, mais bien plutôt l'exploration des modes de relations qui s'établissent entre tous les existants (les humains certes mais aussi tous les non-humains).

Si Latour repère une quinzaine de modes d'existence chez les Modernes (lesquels se croisent et s'entre-croisent débouchant sur plusieurs centaines de registres), il n'entrevoit guère d'autres solutions pour pactiser avec Gaïa que le reflux de l'ontologie naturaliste et des découpages qu'elle impose. Par exemple, évoquant ce qu'il nomme le registre de la " Métamorphose » (un des quinze modes d'existence repérés) il souligne que les "Modernes» ont quelques proximités (quoi qu'ils en pensent) avec les "primitifs » en croyant, tout comme eux, aux entités invisibles qui agissent par-delà les consciences. Ainsi là où les non-Modernes ont leurs esprits, leurs fantômes, leurs êtres surnaturels déposés dans les corps mais aussi dans toutes les sortes d'existants, les "Modernes » ont aussi leurs entités immatérielles, celles qui conduisent au " je est un autre ». Là où les premiers ont leurs chamanes, leurs sorciers, leurs marabouts, les seconds ont leurs psychanalystes, leurs psychothérapeutes. Si les "Modernes » avaient su identifier ces êtres de la métamorphose, les respecter et montrer qu'ils communiquaient avec eux, nul doute qu'ils se seraient trouvées des affinités avec ceux qu'ils tentaient de « civiliser » et peut-être même auraient-ils suscité chez eux un certain intérêt: «Ah, vous aussi vous avez à faire avec ces êtres? ».

Pour le dire dans une sorte de précipité d'analyse, $B$. Latour suggère que l'existence des «Modernes » est déposée dans les modalités de leurs rapports au monde. Ces modalités, malgré leur foisonnement, font peu de cas de tous les existants qui composent Gaïa. Les humains se pensent comme des sujets 
qui asservissent les objets du monde (quand ils ne transforment pas les sujets eux-mêmes en objets) jusqu'à la destruction possible dudit monde. Ainsi ne peuvent-ils penser leur existence que comme le résultat d'une prédation nécessaire de leur environnement. Dès lors que la séparation entre objet et sujet disparaît et que les sujets ne sont que des "quasi-sujets » attachés à un monde d'objets lesquels conditionnent leur existence et que, de la même façon, les objets ne sont plus que des "quasi-objets ", dès lors que ce qui leur donne vie (ou mort) résulte des liens qu'ils ont avec les sujets, alors il n'est plus question de penser l'existence via l'ontologie de l'être ou via la sociologie de l'individu. L'existence ne se perçoit plus au travers d'une interaction entre société et individu mais comme un processus relationnel entre tous les existants du monde ou du cosmos. Dès lors, pour les sciences sociales et plus généralement "les humanités scientifiques», la réflexion sur l'existence suppose un décadrage total.

Descola et Latour, par-delà leurs différences et surtout via leurs convergences, nous invitent à repenser l'existence à partir d'une compréhension des modes relationnels entre tous les existants. A la question que pose l'article de Martucelli «Une sociologie de l'existence est-elle possible? ", sans doute que Descola et Latour pourraient répondre : Oui, à la condition que les humanités scientifiques acceptent la pluralité des ontologies et que soient repensées les modalités de co-existence de tous les existants ! En cela, ces deux auteurs pourraient aisément se retrouver en compagnie d'E. Morin et de sa «pensée complexe » et peut-être même d'A. Piette et de son « anthropologie existentiale » qui se donne à comprendre comme opposée à ce qu'il nomme le « relationnisme».

\section{CONCLURE AVEC MORIN ET PIETTE}

Nous avons jusque-là tenu dans l'ombre des auteurs comme E. Morin ou A. Piette, lequel voit ses travaux largement commentés au sein de ce numéro de la revue Parcours Anthropologiques. Peut-on conclure avec ces deux auteurs sans tenter de marier la carpe et le lapin ? Je peux en faire l'essai.

$\mathrm{Nul}$ doute que la sociologie d'E. Morin peut cousiner sans grande difficulté avec les approches de P. Descola et de B. Latour même si ces deux derniers ne mobilisent jamais ses productions. En effet, depuis de nombreuses années le théoricien de la complexité, plus reconnu en Amérique Latine que par les institutions académiques hexagonales, développe, au travers d'une œuvre monumentale, ce qu'il désigne sous le vocable de pensée complexe. Celle-ci ne peut évidemment pas se résumer en quelques lignes mais ses principales orientations peuvent être rappelées. Selon Morin, rien n'est connaissable sans transdisciplinarité. Physique, biologie, philosophie, sociologie, anthropologie doivent être convoquées pour penser le vivant. Distinguer sans séparer, sans disjoindre telle est l'orientation majeure de la pensée complexe. Le monde, 
l'univers, sont multidimensionnels, ils ne peuvent donc être embrassés dans leur complexité depuis une focale unique. Dans son vocabulaire, le monde humain ne peut être envisagé séparément de la biosphère. Tout ce qui participe de l'existant relève du principe "d'auto-éco-organisation ». Autoproduction et auto-engendrement mêlent, dans un mouvement continu (comme un tourbillon), toutes les entités existantes entre-elles. Il n'y a pas de cause et d'effets mais que des systèmes de rétroaction où des éléments biologiques et culturels s'enchevêtrent. Ainsi, toute lecture (naturaliste) séparant l'être et son environnement et tentant de prouver qu'un des deux éléments peut déterminer ou sur-déterminer l'autre est étrangère à la pensée complexe. Comme chez Descola et Latour, le déplacement analytique proposé pour penser l'existence ne se limite pas à quelques glissements. C'est un changement de paradigme qui est suggéré. Pour sortir par le haut, et non par le chaos, de l'ère de l'anthropocène, et si d'aventure les sciences sociales ET humaines avaient un avis à donner, c'est à repenser la diversité des attachements possibles entre tous les existants du "plurivers » (I. Stengers, 1997) que Morin nous invite.

La démarche d'A. Piette en apparence bien différente n'est peut-être pas si éloignée de l'intention de Morin. S'il n'est pas question pour moi de résumer ce qu'il nomme lui-même une anthroplogie " existentiale ", entendue comme "science empirique et théorique des humains, des individus séparés, de leurs singularités vivantes, existantes, présentes avec leurs particularités... » (Piette, 2016), sa « phénoménographie » distincte de l'ethnographie n'est peut-être pas en rupture totale avec la pensée relationnelle qu'il pourfend. Certes, pour expliquer ce qu'il nomme le "volume d'être, Piette refuse explicitement toute centration sur la relation (que ce soient les «endorelations» ou les «exorelations ») ». Considérant que toutes les sociologies ou anthropologies qui cherchent à expliquer les comportements individuels et collectifs par les trajectoires, les situations, les interactions, les systèmes de relations, les structures mentales... oublient l'essentiel à savoir ce "reste» qui caractérise « l'individu concret existant » avec sa sensibilité irréductible à toute autre (ce « reste » que Jankélévitch aurait sans doute nommé le «je-ne-sais-quoi », cette entité insaisissable qui, selon lui, rendait possible le devenir du vivant), on pourrait estimer que Piette se situe dans un ailleurs théorique assez éloigné d'un Morin (et a fortiori d'un Latour dont il tient à se démarquer explicitement ${ }^{5}$ ). Pourtant, je ne retiens pas cette interprétation. En effet, si le souci fondamental de Piette consiste en une prise en compte de tout ce qui peut être éprouvé par un humain dans la multiplicité des événements et

\footnotetext{
5 Ainsi Gofmann (interaction), Latour (réseaux), Levi-Straus (structure et système), Bourdieu (trajectoire, habitus) mais aussi plus généralement l'ethnographie (analyse de la relation chercheur/acteur) sont désignés par A. Piette comme incarnant les sciences sociales "relationnistes». Selon lui ces différentes approches pour utiles qu'elles soient ne se préoccupent que de l'étude du social et du culturel, laissant de côté l'essentiel à savoir l'existence humaine laquelle se loge en deçà et au-delà de toute dimension relationniste.
} 
instants de son existence, depuis ce "volume d'être unique qui se développe in utero » jusque dans la solitude (même accompagnée) du passage à la mort, si selon lui le spécialiste de "science humaine » (discipline qu'il distingue des sciences sociales) doit s'attacher à capter le maximum de ces instants dans un travail de "filature » aussi permanent que possible de l'individu étudié (tout comme le volcanologue utilise un sismographe pour percevoir tous les instants de vie d'un volcan même lorsque celui-ci "dort ", "l'anthropologue des existences" doit pouvoir s'appuyer sur toute la panoplie technique moderne pour mener à bien ses filatures - caméra, shadowing, puces électroniques...), alors nul doute que cet anthropologue partisan de «l'ontisme réaliste», ce phénoménographe cherchant à saisir le « rayonnement » singulier de chaque personne, « les raies » spécifiques émises par chacun, tombera sur ce que Morin nomme la complexité. Biologie, psychologie, sciences sociales ET humaines, devront s'entremêler, s'entreaider pour dégager (séparer?) l'entrelacs de ce qui relève d'une part du "volume d'être » et d'autre part des relations (endo et exo), des structures mentales... Séparer analytiquement ce qui est mélangé concrètement dans le vivant d'une existence c'est participer de la compréhension de ce mélange. Aussi, tant par-delà les différences de vocabulaire que des programmes de recherche, l'anthropologie des existences de Piette me semble relever de la même intention que celle de Morin (comme de celles de Descola et Latour) : changer de paradigme pour penser le vivant; en somme refaire des sciences sociales ET humaines dans une perspective que la notion d'humanités scientifiques (chère à Latour) me semble pouvoir aussi recouvrir.

\section{BIBLIOGRAPHIE}

Luc BOLTANSKI et Laurent THEVENOT, De la Justification, Les économies de la grandeur, Paris, Gallimard, 1991.

Vincent DE GAULEJAC, Qui est Je, Paris, Seuil, 2009.

Philippe DESCOLA, Par-delà nature et culture, Paris, Gallimard, 2005.

Gilles HERREROS, "Enquêtes sur les modes d'existence des modernes de Bruno Latour", in Revue Française de Sociologie, vol. 55, n 3, 2014.

Jean Paul KAUfMANN, Ego. Pour une sociologie de l'individu. Une autre vision de l'homme et de la construction du sujet, Paris, Nathan, 2001.

Bruno LATOUR, Nous n'avons jamais été modernes, Essai d'anthropologie symétrique, Paris, la Découverte, 1991.

Bruno LATOUR, Petite réflexion sur le culte moderne des dieux faitiches, Paris, Synthélabo, 1996.

Bruno LATOUR, Changer la société, refaire de la sociologie, Paris, La Découverte, 2006.

Bruno LATOUR, Cogitamus, Paris, la Découverte, 2010. 
Bruno LATOUR, Enquête sur les modes d'existence. Une anthropologie des Modernes, Paris, la Découverte, 2012.

Danilo MARTUCELLI et François DE SINGLY, Les sociologies de l'individu, Paris, Armand Collin, 2009.

Danilo MARTUCELLI, «Une sociologie de l'existence est-elle possible ? », SociologieS [En ligne]. URL : https://journals.openedition.org/sociologies/3617

George Herbert MEAD, L'esprit, le soi et la société, Paris, PUF, 1963.

Edgar MORIN, La méthode, Tome1, La nature de la nature, Paris, Seuil, 1977.

Edgar MORIN, La méthode, Tome 2, La vie de la vie, Paris, Seuil, 1980.

Edgar MORIN, La méthode, Tome 3, La connaissance de la connaissance, Paris, Seuil, 1986.

Edgar MORIN, La méthode, Tome 4, Les idées, leurs habitants, leur vie, leurs moeurs, leur organisation, Paris, Seuil, 1991.

Edgar MORIN, La méthode, Tome 5, L'humanité de l'humanité, Paris, Seuil, 2001.

Edgar MORIN, La méthode, Tome 6, L'éthique, Paris, Seuil, 2004.

Albert PIETTE, «De la relation à l'existence: sciences sociales ou sciences humaines?", Revue du Mauss, 2016/1, n 47, 2016.

Isabelle STENGERS, Cosmopolitiques, 7 volumes, Paris, Synthélabo, 1997.

Alain TOURAINE, Critique de la modernité, Paris, Fayard, 1992.

RÉSUMÉ: Les sociologues mobilisent abondamment depuis plusieurs décennies les notions d'acteur, de sujet, d'égo, de «je», mais le plus souvent ils laissent de côté la question de l'existence. Lorsqu'ils s'essaient à l'approcher (Martucelli), le détour par la philosophie leur est indispensable mais il n'est pas certain que leur lecture des philosophes permette toujours de dégager une solide "plus-value cognitive ». Il est suggéré ici de faire un pas de côté en s'intéressant à la multiplicité des existants et à la pluralité de leurs modes $\mathrm{d}$ 'attachements (Descola et Latour), tenant ainsi en lisière le relationnisme (que pourfend Piette) et le recours à une ontologie radicale.

MOTS-CLÉS : Anthropocène, existants, existence, modes d'existence, ontologie, paradigme. 